\title{
[0001]-Oriented InN Nanoleaves and Nanowires: Synthesis, Growth Mechanism and Optical Properties
}

\author{
Min Liu ${ }^{1} \cdot$ Hui-Qiang $\mathrm{Liu}^{2} \cdot$ Sheng $\mathrm{Chu}^{2} \cdot$ Ru-Fang Peng ${ }^{1} \cdot$ Shi-Jin Chu ${ }^{1}$
}

Received: 21 February 2016/Revised: 5 May 2016/Published online: 11 July 2016

(C) The Chinese Society for Metals and Springer-Verlag Berlin Heidelberg 2016

\begin{abstract}
Novel indium nitride ( $\mathrm{InN}$ ) leaf-like nanosheets and nanowires have been grown on Si substrate by chemical vapor deposition method. The characterization results indicate that the samples are single-crystalline, and the growth direction of the nanowires and nanoleaves is [0001]. The growth mechanism of the $\mathrm{InN}$ nanoleaves is following the pattern of vapor-liquid-solid process with a three-step growth process. In addition, the room temperature photoluminescence spectra of two nanostructures show band-to-band emissions around $0.706 \mathrm{eV}$, where the emission from single nanoleaf is stronger than nanowire, showing potential for applications in optoelectronic devices.
\end{abstract}

KEY WORDS: Novel indium nitride (InN); Chemical vapor deposition (CVD); Nanoleaf; Crystal structure; Photoluminescence (PL)

\section{Introduction}

As representative group III-V nitride semiconductors [1], $\mathrm{InN}$ materials are important for their interesting properties, such as high electrical conductivity and electron mobility [2]. In addition to thin film-type InN materials, the research on InN nanostructures has attracted much attention for their potential for advanced applications in the nanoscale devices with feasible tunability of properties, applied in highspeed field effect transistors (FETs) [3], biosensors [4],

Available online at http://link.springer.com/journal/40195

Sheng Chu

chusheng@mail.sysu.edu.cn

$\triangle$ Ru-Fang Peng

pengrufang@swust.edu.cn

1 State Key Laboratory Cultivation Base for Nonmetal Composites and Functional Materials, Southwest University of Science and Technology, Mianyang 621010, China

2 State Key Laboratory of Optoelectronic Materials and Technology, Sun Yat-Sen University, Guangzhou 510275, China electrochemical sensor [5], high-output piezoelectric nanogenerators [6] and terahertz spectroscopy [7].

The properties of nanostructures usually depend on its size and shape. Various InN nanostructures, including onedimensional (1D) nanotubes, two-dimensional (2D) nanosheets and three-dimensional (3D) nanoflowers, have been synthetized by different physical and chemical methods [8-15]. Reports on the ascendant InN epitaxial nanocolumns [15] were synthesized by controlling two factors of various substrate temperatures and III/V ratios, indicating the further development of the $2 \mathrm{D} \mathrm{InN}$ nanostructures. For 2D nanomaterials, those with leaf-like morphology represent a special group of nanostructures, like $\mathrm{GaN}$ and $\mathrm{ZnO}$ hexagonal nanoplates, with potential in optical application [16-21]. For instance, symmetric nanoplates are excellent source for whispering gallery mode nanolaser resonators [22, 23], and nanodisks or nanobelts are widely used as gas sensors and photodetectors $[24,25]$. Nevertheless, studies of the 2D structure of InN nanomateirals are scarce so far. Previously we have demonstrated on the growth and characterization of two kinds of InN nanomaterials (nanowires and nanonecklaces) by a simple catalytic vapor deposition technique [26]. 
Here in this present study, we have synthesized InN leaf-like nanosheets and nanowires via the cost-effective, clean and rapid CVD method [26]. The morphology, composition and microstructure of the products were systematically characterized. The growth mechanism of $\mathrm{InN}$ nanoleaves materials was analyzed and discussed in detail. The three-step growth mechanism was presented based on temperature-dependent growth observation. Furthermore, room temperature photoluminescence (PL) spectra of two $\mathrm{InN}$ nanostructures demonstrated fairly good optical properties.

\section{Experimental}

The single-crystal $\mathrm{InN}$ nanoleaves reported here were grown by CVD method in the presence of gold colloid (BBI solution, thickness: $60 \mathrm{~nm}$ ) as the catalyst. The raw materials were pure In powder (99.999\%, Xiya reagent) and $\mathrm{NH}_{3}$ gas (99.99\% purity, Messer). The p-type silicon (100) substrate coated with gold colloid was placed facedown above the indium powders loaded in the quartz boat, and the whole facilities were placed at the central heating zone of tubular resistance furnace. Before heating, the quartz tube was pumped to around $3 \mathrm{~Pa}$ and flushed several times with $\mathrm{Ar}$ (carrier gas: $99.999 \%$ purity) to remove impurities. Then, the center of the furnace was heated up to the reaction temperature of $700{ }^{\circ} \mathrm{C}$ with $\mathrm{Ar}$ and kept at this temperature for 100 min under $\mathrm{NH}_{3}$ with a flow rate of $150 \mathrm{sccm}$. Finally, the furnace was naturally cooled down to room temperature with Ar, and gray and black-colored products were formed on the silicon substrate.

The morphological and microstructural properties of the as-synthesized products were characterized by field emission scan electron microscopy (SEM; Model UItra 55) equipped with energy dispersive X-ray spectroscopy (EDS), X-ray diffraction (XRD) on a Philips X' Pert PRO diffractometer equipped with $\mathrm{Cu} \quad K_{\alpha}$ radiation $(\lambda=0.15418 \mathrm{~nm})$, transmission electron microscopy (TEM; Model Libra $200 \mathrm{PE}$, operated at $200 \mathrm{kV}$ ), and room temperature PL spectrum (model; in Via) carried out on a Raman microscope using a $514 \mathrm{~nm} \mathrm{He}-\mathrm{Cd}$ laser as the excitation source.

\section{Results and Discussion}

Figure 1a, b shows the typical SEM images of $\mathrm{InN}$ nanostructures in low and high magnification, respectively. It can be clearly seen that the samples are mainly composed of two kinds of $\mathrm{InN}$ nanostructures: nanowires and nanoleaves. The typical length and diameter of the individual nanowires range from 3 to $10 \mu \mathrm{m}$ and 80 to $150 \mathrm{~nm}$.
Highly magnified side-view SEM image of a single nanoleaf reveals that the nanoleaves are with a lateral size of $(15 \pm 0.5) \mu \mathrm{m}$ and a thickness of $100 \mathrm{~nm}$, showing a novel corn leaf-like structure (Fig. 1c). Undoubtedly, the nanoleaves are of thin thickness and flat surface, which has not been reported before. In addition, the co-existence of different morphology nanomaterials indicates that the CVD growth of various nanostructures may have a large interval range in growth process. The vapor-liquid-solid (VLS) growth mechanism [27] is promoted by the existence of gold nanoparticles; Au acts as a catalyst in order to reduce the activation energy for develop nucleation position greatly.

Figure 2 displays in more detail typical XRD patterns of InN nanostructures. All of the strong diffraction peaks in the spectrum can be indexed to hexagonal wurtzite structure InN (JCPDS card No. 50-1239, $a=0.353 \mathrm{~nm}$, and $c=0.57 \mathrm{~nm}$ ). It is in good agreement with the previously reported values of InN bulk crystals [28]. The full-width at half-maximum (FWHM) of those peaks is keen-edged, indicating large-sized single crystal. There are some characteristic peaks of other phases ( $\mathrm{Si}, \mathrm{Au}$, oxides) detected in XRD. The O-related peaks originated from the native oxidation of substrate or the formation of indium oxides potentially. To clearly identify the appearance of oxide peaks, we conclude that the diffraction peaks about oxides slightly match with the reported $\mathrm{In}_{2} \mathrm{O}_{3}$ nanostructure [29] (JCPDS card No. 06-0416) with cubic system. Simultaneously, we can see the (100) diffraction peak appeared which belongs to InN nanostructure, and the (002) diffraction peak at $31.330^{\circ}$ and the (101) diffraction peak at $33.156^{\circ}$ are closer to $\operatorname{InN}$ but $\operatorname{In}_{2} \mathrm{O}_{3}$, where the two peaks' relative intensities are in accordance with $\mathrm{InN}$ crystal with hexagonal wurtzite structure. Therefore, the related $\mathrm{O}$ peaks might be the introduced impurities and the native $\mathrm{SiO}_{2}$ formed on $\mathrm{Si}$ substrate.

The EDS measurements were carried out to analyze the chemical composition of the samples. Figure 3a illustrates a representative SEM image of the part of the sample that contains nanowires and nanoleaves, while the EDS spectrum of the marked position in Fig. 3a is shown in Fig. 3b. The EDS spectrum contains six elements: C, N, O, In, Si and $\mathrm{Au}$, and the atomic ratio of In: $\mathrm{N}$ is close to $1: 1$, suggesting a good stoichiometry of the materials. While the silicon peak originates from the substrate, carbon and oxygen peaks are derived from the introduction of the gas outside or oxidation on the substrate [17].

Figure $4 a-d$ clearly shows the TEM images of individual $\mathrm{InN}$ nanowire and nanoleaf. Figure $4 \mathrm{a}$ shows a typical TEM image of single nanowire with a smooth morphology. A high-resolution TEM (HRTEM) image (Fig. 4b) reveals that the length growth direction of InN nanowire is [0001] with the lattice spacing of $0.281 \mathrm{~nm}$, which is in good 

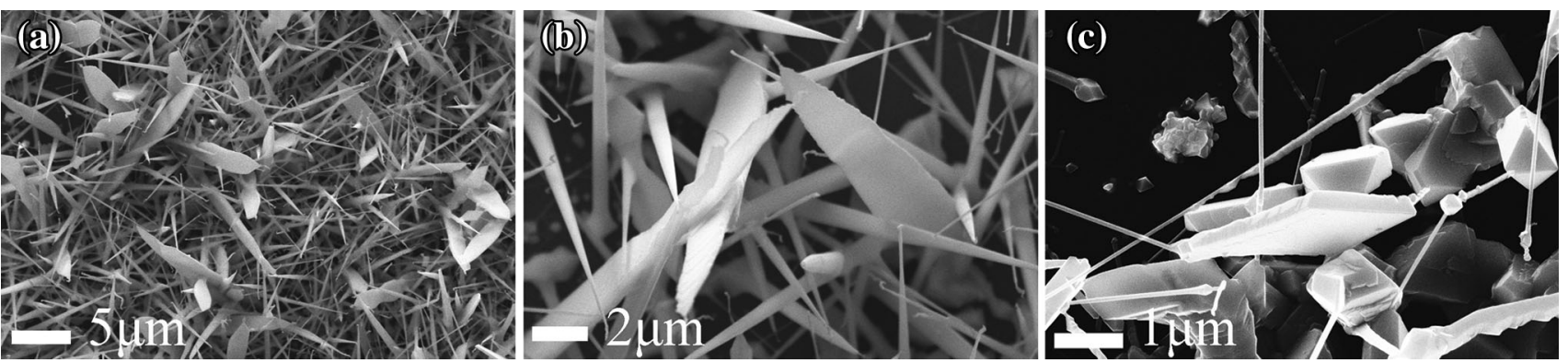

Fig. 1 a, b Less and highly magnified SEM images of the nanoleaf and nanowire structures; c side-view of highly magnified SEM image

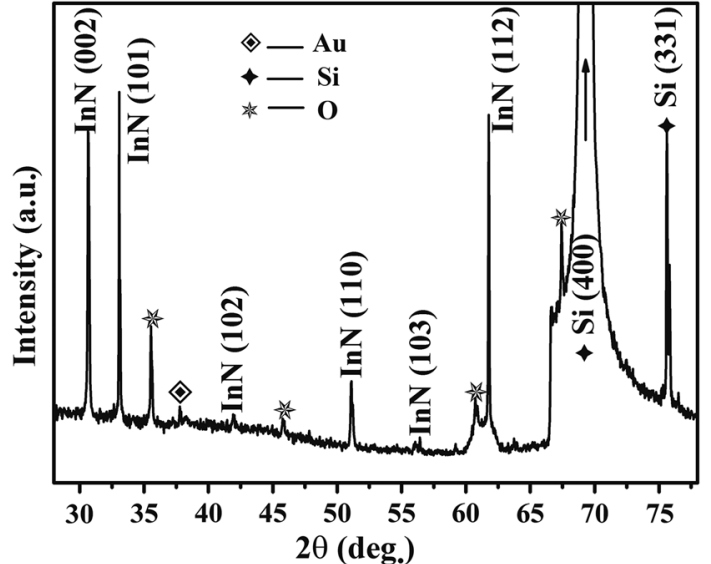

Fig. 2 XRD pattern of the InN nanostructure

agreement with the (100) planes of InN hexagonal structure correspondingly [30, 31]. The inset in Fig. $4 \mathrm{~b}$ is the selected area electron diffraction (SAED) pattern from the corresponding area of nanowire, confirming that the product is single-crystalline and grows directly along the [0001] direction. Figure 4c presents the TEM image of InN nanoleaf showing clearly the round head at the tip of the nanoleaf. Figure 4d shows a lattice-resolved TEM image taken from the edge region of the nanoleaf, and the measured interplanar spacing of the crystalline phase is $0.284 \mathrm{~nm}$, which corresponds to the (101) plane of the hexagonal InN. Synchronously, the illustration of the Fig. $4 \mathrm{~d}$ shows the HRTEM pattern of the edge region of the nanoleaf. It exhibits a spot pattern of a single-crystal hexagonal phase, no grain boundary was found in the sample, and the length direction of InN nanoleaf is [0001]. Both the HRTEM and fast Fourier transformed (FFT) results demonstrate that the nanoleaves are single crystals with a hexagonal structure. For the purpose of studying the native structure, the atomic accumulation structure model of InN nanoleaves is described in Fig. 4e, f. As is shown in Fig. 4f, the growth direction of nanoleaves is [0001], with the two polar side surfaces being $\{10 \overline{1} 1\}$ and $\{10 \overline{1} \overline{1}\}$. By using the standard lattice parameters of $\mathrm{w}-\mathrm{InN}$, the angle between two corresponding facets on the adjacent facets of leaf-like structure is calculated to be $116^{\circ}$ [26], which is closed to the actual measurement of $117.7^{\circ}$ (Fig. 4c).

Based on the above results, the leaf-like InN nanostructure exhibits VLS growth mechanism, which is different from the products prepared by solvent method. So far, most of researches about nanomaterials with the growth mechanism of VLS pay attentions to the one-dimensional nanomaterials [32], such as nanowires and nanorods. These nanomaterials show two typical characteristics: (1) the liquid metal catalyst act as the nucleation point of the absorption gas reaction and (2) the metal catalyst particles can be observed at the top of the nanomaterial. As shown in Fig. 5a, b, the metal particles are at the end of nanoleaf and nanowire, which indicates the presence of Au particles. Besides, Au particles are easy to combine with In droplets. Consequently, the size of Au particles has influence on the size of nucleation sites and the surface area of the gas absorption. Therefore, the gold catalyst plays a key role in the growth of the leaf-like nanostructure.

A series of experiments were carried out to study the growth process in detail, and we have recorded the morphology of InN nanostructures at different growth temperatures. As shown in Fig. 6a-d, during the whole growth process of the InN nanostructures, we can conclude that the size of the nanoleaf increase as the temperature increases continuously from 500 to $650{ }^{\circ} \mathrm{C}$. There are obviously three steps to the growth process of $\mathrm{InN}$ nanostructures as shown in Fig. 6e. The first step is the formation of nucleation particles. Indium atoms and ammonia molecules reacted and nucleate with the assistance with gold catalyst and nanowire-like structures start to grow (Fig. 6a). The next step is the growth of nanowires. The arrival of new In and $\mathrm{N}$ atoms can be repeated to form new InN layer in the growth direction, which is explained schematically in Fig. $6 \mathrm{~b}$, and it is quite clear that the $\mathrm{InN}$ nanowire is followed by the VLS growth mechanism [33]. The last step is the formation of leaf-like InN morphology. This process is embodied through Fig. 6c, d. Based on all above-related process, the growth procedure can be concluded by Fig. 6e. In order to understand the mechanism of $\mathrm{InN}$ nanoleaf 


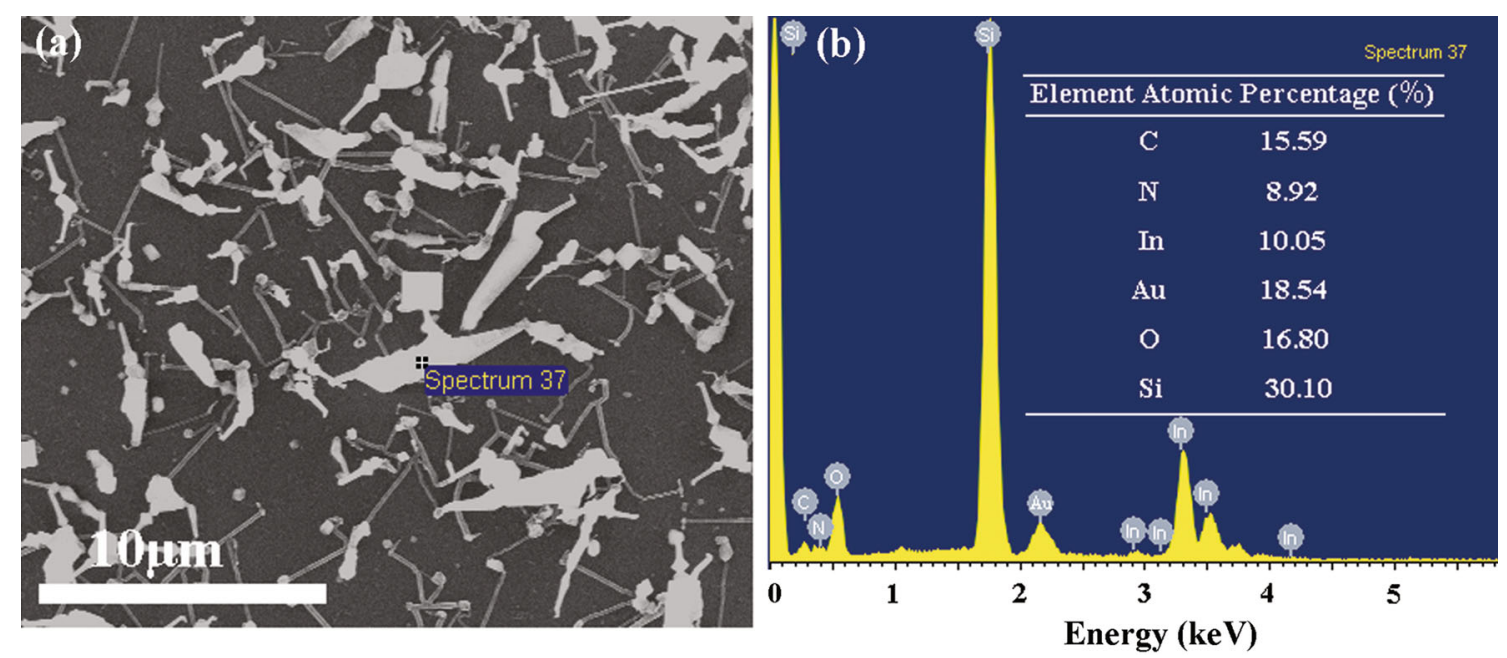

Fig. 3 SEM image of the as-grown InN nanoleaves a and EDS spectrum corresponding to the spot marked spectrum $37 \mathbf{b}$

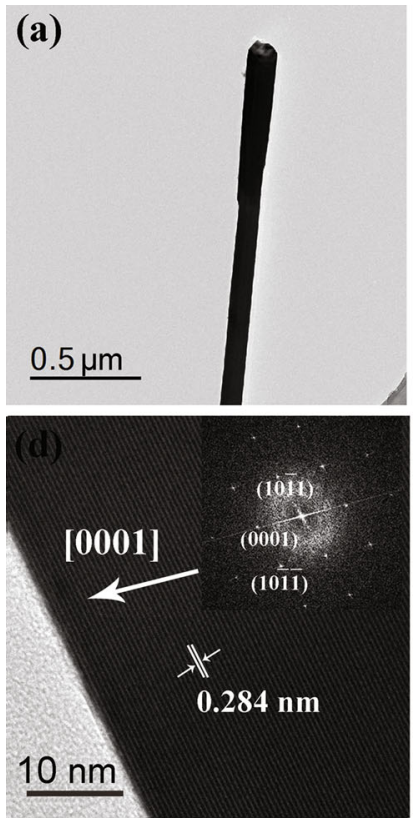

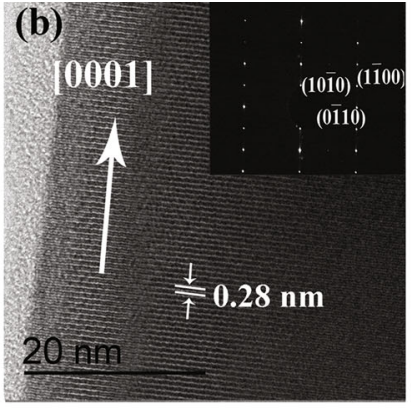

(e)

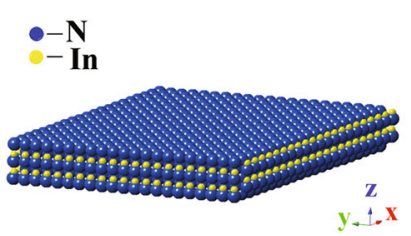

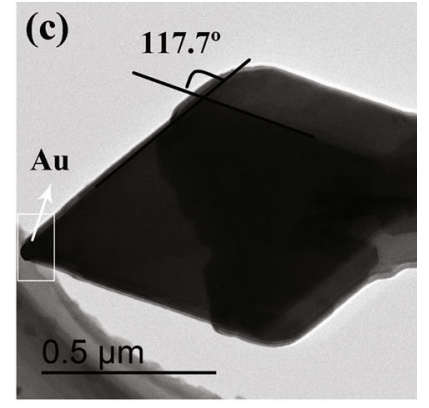

(f)

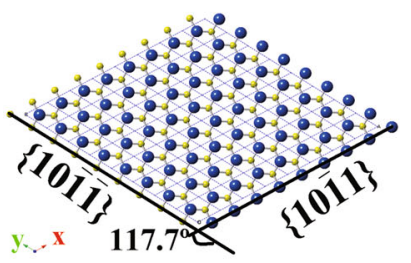

Fig. 4 a TEM image of the individual nanowire, $\mathbf{b}$ corresponding HRTEM image and SAED pattern (the inset) of the nanowire, $\mathbf{c}$ TEM image of the individual nanoleaf, d corresponding HRTEM image and FFT pattern (the inset) of the nanoleaf, e model of InN atomic accumulation structure, $\mathbf{f}$ crystal structure of InN nanoleaf, showing the positively or negatively charged $\{10 \overline{1} \overline{1}\}$ and $\{10 \overline{1}\}$ planes

deeply, the size of (0001) surface was gradually decreased and the side facets of $\{10 \overline{1} 1\}$ and $\{10 \overline{1} \overline{1}\}$ planes appeared to form the leaf-like structure. Because the terminated $\mathrm{In}^{3+}-\{10 \overline{1} \overline{1}\}$ and $\mathrm{N}^{3-}-\{10 \overline{1} 1\}$ planes had possessed finite charges and dipole moments [26], they would result in the positive and negative electrostatic surface energy increase. To keep the structure stable, the total surface free energy including the surface energy and the electrostatic surface energy should be minimized, when the $\{10 \overline{1} 1\}$ and $\{10 \overline{1} \overline{1}\}$ planes continue to crystallize with increasing electrostatic surface energy, but the $\{10 \overline{1} 1\}$ and $\{10 \overline{1} \overline{1}\}$ side facets have opposite termination to keep the conservation of energy. The alternation of the dipole moments and the change of the electrostatic surface energy will lead to repeated formation of $\operatorname{In}^{3+}-\{10 \overline{1} \overline{1}\}$ and $\mathrm{N}^{3-}-\{10 \overline{1} 1\}$ side facets along the $c$-axis direction and finally produce the $\mathrm{InN}$ nanoleaf. Simultaneously, it also can be concluded that the longitudinal growth influences the lateral growth process. An axial perturbation due to fluctuation of growth conditions may cause the bulging of middle region of the structures and result in a leaf-like InN morphology. In the whole growth process, literature data of the Gibb's free energy for the 

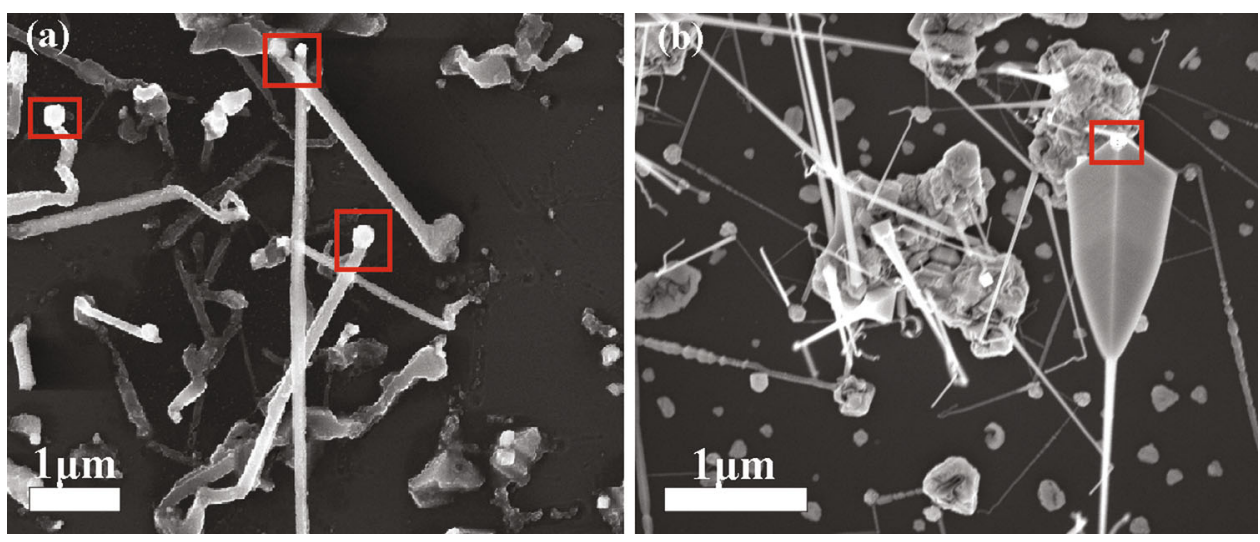

Fig. 5 SEM images of InN nanowires a and InN nanoleaf $\mathbf{b}$ based on growth mechanism
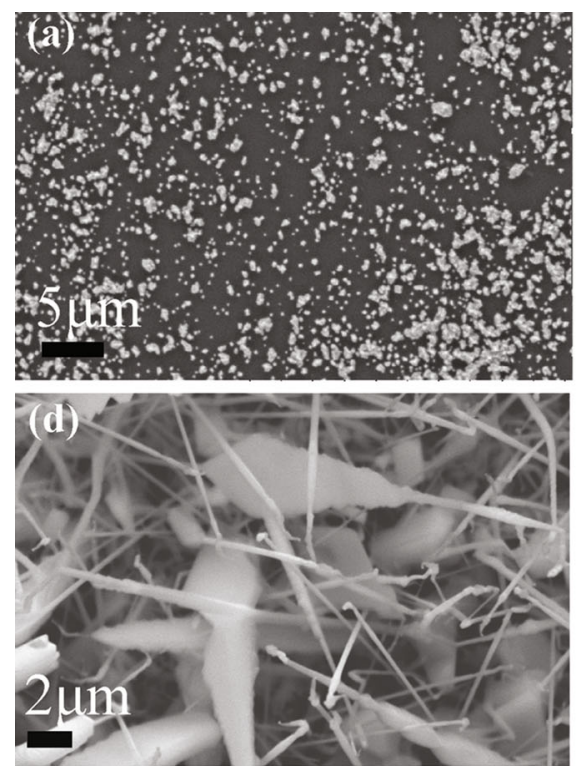

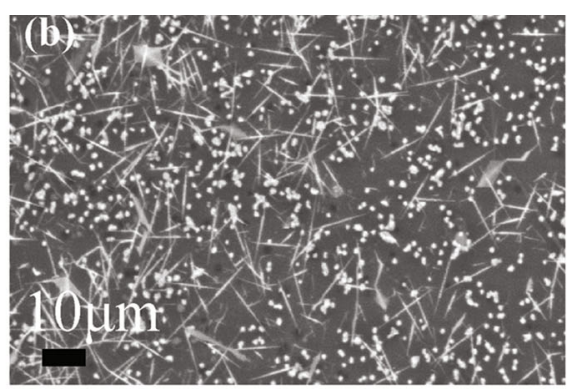

(e)
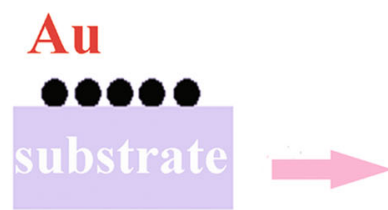

\section{In $\mathrm{N}$ nanowire}

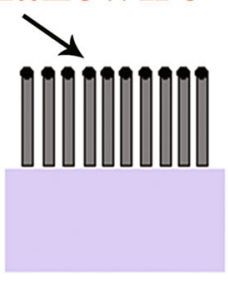

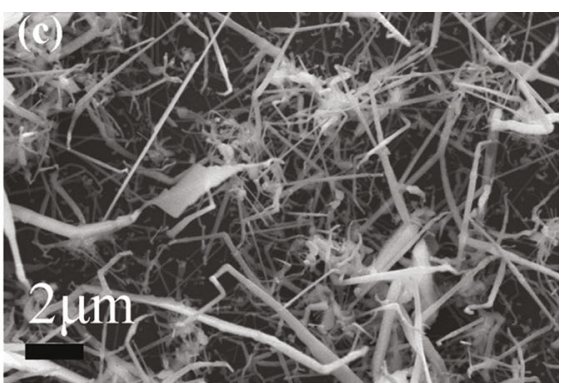

Nanoleaf growth

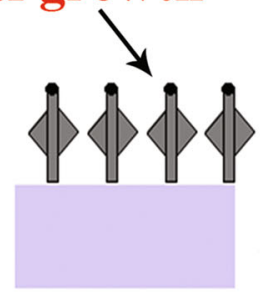

$\rightarrow$

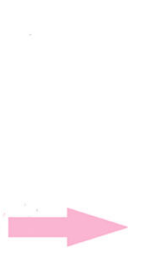

Fig. 6 Whole growth process of InN nanowires and nanoleaves at different growth temperatures keeping in accordance with the same growth conditions: a $500{ }^{\circ} \mathrm{C}, \mathbf{b} 550{ }^{\circ} \mathrm{C}$, c $600{ }^{\circ} \mathrm{C}, \mathbf{d ~} 650{ }^{\circ} \mathrm{C}$, e schematic diagram of InN nanostructure growth in Si (P type: 100) substrate

formulation of $\mathrm{InN}$, under conditions close to ours, is listed as follows [34]:

$\mathrm{In}+\mathrm{NH}_{3} \rightarrow \mathrm{InN}+32 \mathrm{H}_{2} \quad G=-86 \mathrm{~kJ} / \mathrm{mol}$.

Figure 7a shows the room temperature PL spectrum of the nanowire and nanoleaf. The PL peak emission is located at around $0.706 \mathrm{eV}$ which is attributed to Mahan excitonic recombination [35]. The band-to-band Mahan emission transition was in agreement with that of the highquality nanorods and epitaxial films, which is also consistent with that measured in n-type degenerate $\mathrm{InN}$ [36]. In addition, the FWHM of the band-to-band emission peak of the single nanowire and nanoleaf are 26.14 and $25.17 \mathrm{meV}$, respectively, indicating that these two nanostructures have high crystalline quality. Otherwise, the FWHM of nanoleaf is lower than that of nanowire, showing a higher degree of crystallinity. Notably, the emission intensity from the nanoleaf is stronger due to the fact that the nanoleaf is with good crystallinity [37] and generates the effect of multi-scattering inside itself with the average crystalline size of leaf-like structure greatly larger than nanowire, thus resulting in the high recombination probability at band edge region being improved. In addition, the temperature-dependent PL spectra of InN nanoleaf are shown in Fig. 7b, indicating a weak shift (peak position) from $0.706 \mathrm{eV}$ at $180 \mathrm{~K}$ to $0.698 \mathrm{eV}$ at $300 \mathrm{~K}$. Simultaneously, a shoulder is weakly visible at $\sim 64 \mathrm{meV}$ below the main transition due to the coupling of longitudinal (LO) phonons [35]. The thermal variation of the band gap energy can be calculated to be $8 \mathrm{meV}$ as a result of the lattice expansion and electron-phonon interaction [38] and broadens with increasing 

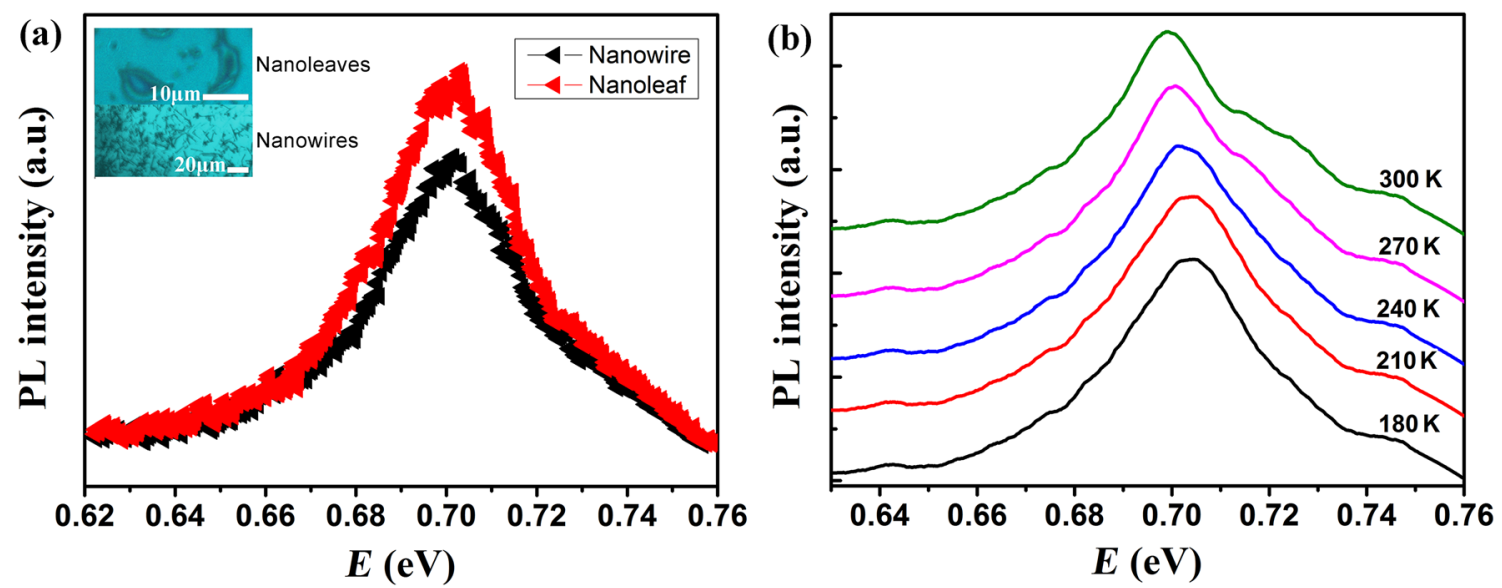

Fig. 7 a PL spectra of nanoleaf and nanowire at room temperature $(300 \mathrm{~K})$, b temperature-dependent PL spectra of nanoleaf

temperature. This line-shape analysis confirms that the emission of nanoleaf is mainly due to the Mahan excitation. Consequently, these high-quality $\mathrm{InN}$ crystallites have potential applications in optoelectronics, and may be widely used in IR nanocavity lasing.

\section{Conclusion}

Two different kinds of $\mathrm{InN}$ nanostructures including nanoleaves and nanowires were synthesized by CVD. The synthesis of high-purity single-crystalline InN nanostructures with hexagonal wurtzite crystalline was obtained. The nanowire and nanoleaf growth were preferentially along [0001] direction. The nanowire and leaf-like structures grew along its axial direction via the VLS mechanism. The room temperature PL spectra of these InN nanostructures demonstrated the emission peak around $0.706 \mathrm{eV}$. These results demonstrate that leaf-like $\mathrm{InN}$ nanosheets are promising for near-infrared optoelectronics applications.

Acknowledgments This work is supported by the National Natural Science Foundation of China (No. 51572230), the National Defense Fundamental Research Projects (No. A3120133002), the Youth Innovation Research Team of Sichuan for Carbon Nanomaterials (No. 2011JTD0017), the Applied Basic Research Program of Sichuan Province (No. 2014JY0170) and the Postgraduate Innovation Fund Project by Southwest University of Science and Technology (No. 15ycx007) and the Open Project of State Key Laboratory Cultivation Base for Nonmetal Composites and Functional Materials (No. 13zxfk09).

\section{References}

[1] X.B. Wang, J.H. Song, F. Zhang, C.Y. He, Z. Hu, Z.L. Wang, Adv. Mater. 22, 2155 (2010)

[2] P. Siddiqua, W.A. Hadi, M.S. Shur, S.K. O’Leary, J. Mater. Sci. Mater. Electron. 26, 4475 (2015)
[3] M. Oseki, K. Okubo, A. Kobayashi, H. Fujioka, Sci. Rep. 4, 3951 (2014)

[4] E. Óscar, B.N. Fernado, D.H. Natalia, V.F. Sirona, M.C. Navarrete, G.C. Agustin, Sens. Actuators B 158, 372 (2011)

[5] N. Sofikiti, N. Chaniotakis, J. Grandal, M. Utrera, M.A. Sánchez-García, E. Calleja, Mater. Lett. 64, 1332 (2010)

[6] C.T. Huang, J. Song, C.M. Tsai, W.F. Lee, D.H. Lien, Z.Y. Gao, Y. Hao, L.J. Chen, Z.L. Wang, Adv. Mater. 22, 4008 (2010)

[7] H. Ahn, C.C. Yu, P. Yu, J. Tang, Y.L. Hong, S. Gwo, Opt. Express 20, 769 (2012)

[8] C.K. Chao, J.I. Chyi, C.N. Hsiao, C.C. Kei, S.Y. Kuo, H.S. Chang, T.M. Hsu, Nanotechnology 17, 3930 (2006)

[9] M. Amirhoseiny, S.S. Ng, Z. Hassan, Mater. Sci. Semicond. Process. 35, 216 (2015)

[10] Z.X. Bi, R. Zhang, Z.L. Xie, X.Q. Xiu, Y.D. Ye, B. Liu, S.L. Gu, B. Shen, Y. Shi, Y.D. Zheng, Mater. Lett. 58, 3641 (2004)

[11] T.T. Kang, X.L. Liu, R.Q. Zhang, W.G. Hu, G.W. Cong, F.A. Zhao, Q.S. Zhu, Appl. Phys. Lett. 89, 071113 (2006)

[12] H.L. Xiao, X.L. Wang, J.X. Wang, N.H. Zhang, H.X. Liu, Y.P. Zeng, J.M. Li, Z.G. Wang, J. Cryst. Growth 276, 401 (2005)

[13] A.T.M. Golam Sarwar, S.D. Carnevale, T.F. Kent, M.R. Laskar, B.J. May, R.C. Myers, J. Cryst. Growth 428, 59 (2015)

[14] S. Zhao, S. Fathololoumi, K.H. Bevan, D.P. Liu, M.G. Kibria, Q. Li, G.T. Wang, H. Guo, Z. Mi, Nano Lett. 12, 2877 (2012)

[15] K. Wang, T. Araki, T. Yamaguchi, Y.T. Chen, E. Yoond, Y. Nanishib, J. Cryst. Growth 430, 93 (2015)

[16] C.Z. Zhang, H. Gao, D. Zhang, X.T. Zhang, Chin. Phys. Lett. 25, 302 (2008)

[17] L.Z. Liu, Y.Q. Chen, L.L. Guo, T.B. Guo, Y.Q. Zhu, Y. Su, C. Jia, M.Q. Wei, Y.F. Cheng, Appl. Surf. Sci. 258, 923 (2011)

[18] C.C. Zhao, A.Q. Chen, X. Ji, Y. Zhu, X.C. Gui, F. Huang, Z.K. Tang, Mater. Lett. 154, 40 (2015)

[19] X. Xie, G.Z. Wang, Z.B. Shao, D.P. Li, J. Phys. Chem. C 113, 14633 (2009)

[20] M.J. Chithra, M. Sateya, K. Pushpanathan, Acta. Metall. Sin. (Engl. Lett.) 28, 394 (2015)

[21] Y.L. Zhao, Z. Chen, J. Long, T. Yang, Acta. Metall. Sin. (Engl. Lett.) 27, 81 (2014)

[22] A. Aubry, D.Y. Lei, S.A. Maier, J.B. Pendry, ACS Nano 4, 3293 (2011)

[23] R. Robert, P.H.S. Themistoklis, T. Christian, C. Silke, C. Silke, F.O. Rupert, R. Carsten, Nano Lett. 15, 4637 (2015)

[24] J.Z. Liu, M.Z. Zhong, J.B. Li, A.L. Pan, X.L. Zhu, Mater. Lett. 148, 184 (2015)

[25] S.J. Young, Y.H. Liu, Microelectron. Eng. 148, 14 (2015) 
[26] H.Q. Liu, S. Chu, R.F. Peng, M. Liu, Z.X. Chen, B. Jin, S.J. Chu, Cryst. Eng. Commun. 17, 4818 (2015)

[27] J.Y. Li, J. Liu, L.S. Wang, R.P.H. Chang, Inorg. Chem. 47, 10325 (2008)

[28] C.H. Liang, L.C. Chen, J.S. Hwang, Y.F. Chen, Appl. Phys. Lett. 81, 22 (2002)

[29] S. Zhang, P. Song, H.H. Yan, Z.X. Yang, Q. Wang, Appl. Surf. Sci. 378, 443 (2016)

[30] S.K. Lim, S. Crawford, G. Haberfehlner, S. Gradecak, Nano Lett. 13, 331 (2013)

[31] A.M. Morales, C.M. Lieber, Science 279, 208 (1998)

[32] S.C. Shi, C.F. Chen, G.M. Hsu, J.S. Hwang, S. Chattopadhyay, Z.H. Lan, K.H. Chen, L.C. Chen, Appl. Phys. Lett. 87, 203103 (2005)

[33] X. Ji, S. Cheng, H.R. Hu, H.J. Li, Z.G. Wu, P.X. Yan, AIP Adv. 2, 022150 (2012)
[34] Z.H. Lan, W.H. Wang, C.L. Sun, S.C. Shi, C.W. Hsu, T.T. Chen, K.H. Chen, C.C. Chen, Y.F. Chen, L.C. Chen, J. Cryst. Growth 269, 87 (2004)

[35] M. Feneberg, J. Däubler, K. Thonke, R. Sauer, Phys. Rev. B 77, 245207 (2008)

[36] A. Chandolu, D.Y. Song, M.E. Holtz, I. Gherasoiu, S.A. Nikishin, A. Bernussi, M.W. Holtz, J. Electron. Mater. 38, 557 (2009)

[37] M.C. Johnson, C.J. Lee, E.D. Bourret-Courchesne, S.L. Konsek, S. Aloni, W.Q. Han, A. Zettl, Appl. Phys. Lett. 85, 5670 (2004)

[38] D.Y. Song, M.E. Holtz, A. Chandolu, A.A. Bernussi, S. Nikishin, M. Holtz, L. Gherasoiu, Appl. Phys. Lett. 92, 121913 (2008) 